\title{
Repenser les oncogènes en 1995 ?
}

\author{
Bernard Vandenbunder
}

La découverte du premier oncogène en 1975 et les résultats expérimentaux qui se sont accumulés pendant les années suivantes ont permis l'élaboration d'une théorie unificatrice de la genèse des cancers. Selon cette théorie, les cancers sont dus à l'expression déréglée ou à une mutation d'un nombre limité de gènes appelés oncogènes. Suite à l'élargissement du premier cercle à de nouveaux membres, parents et associés, le statut d'oncogène, et celui d'anti-oncogène, a perdu aujourd'hui sa visibilité. Pour comprendre le rôle de ces acteurs moléculaires, il faut les replacer dans leur contexte, entreprendre une analyse cinétique de leurs interactions, accorder une plus grande place aux données quantitatives. Il faut aussi construire une plate forme conceptuelle cohérente qui permette de confronter les résultats expérimentaux à des modèles théoriques.

\section{TIRÉS À PART}

B. Vandenbunder. l y a vingt ans, l'étude du génome d'un rétrovirus aviaire, le virus du sarcome de Rous, permettait l'identification du premier oncogène viral v-src et du proto-oncogène c-src dont il dérivait [1]. Cette découverte marquait l'irruption de la biologie moléculaire dans la cancérologie. Elle illustrait aussi pour la première fois l'intérêt de l'étude de systèmes "simples", phylogéniquement éloignés pour notre compréhension des mécanismes du cancer, chez l'homme. Transporté par l'enthousiasme de sa découverte, un de ses auteurs écrivait, dans Scientific American, que "les oncogènes sont les gènes qui créent le cancer" [2].

Pendant les années qui ont suivi, on pu assister à la convergence de différentes stratégies de recherche, de différentes conceptions de la genèse des cancers, autour du concept d'oncogène. Il semble, cependant, que la réconciliation autour de ce concept n'ait pas résisté à l'allongement continuel de la liste des gènes impliqués dans le processus cancéreux

\section{qre période : 1975-1985. Une théorie unificatrice?}

Selon la façon dont est abordée l'étude des mécanismes moléculaires du cancer, plusieurs définitions de la notion d'oncogène sont possibles. Les oncogènes viraux sont responsables $\mathrm{du}$ pouvoir pathogène des virus transformants et les gènes cellulaires dont dérivent les oncogènes rétroviraux ont constitué le premier cercle des oncogènes. Certains oncogènes ont été identifiés dans l'ADN extrait de tumeurs parce qu'ils étaient ca- pables d'induire, après transfection dans des fibroblastes, un phénotype transformé, caractérisé par des changements morphologiques, par la capacité de proliférer en gel d'agar mou et de former des tumeurs chez la souris. Enfin, les gènes situés aux points de translocation chromosomique, les gènes mutés ou amplifiés ont pu être appelés oncogènes dans la mesure où les anomalies qui les affectaient se retrouvaient systématiquement dans certains types de tumeurs.

Il y a dix ans, ces différentes voies d'investigation convergeaient de façon remarquable, sur des oncogènes comme c-myc ou c-ras. Puisque ces gènes répondaient aux trois définitions rappelées ci-dessus, on pouvait considérer qu'ils jouaient un rôle crucial dans le processus de cancérisation. L'expression ou l'activation de ces oncogènes était associée à la prolifération des cellules mises en culture. En outre, l'étude biochimique des produits des oncogènes alors identifiés révélait qu'ils étaient tous des éléments des voies de signalisation, facteurs de croissance, récepteurs pour ces facteurs, protéines membranaires associées à ces récepteurs, tyrosine ou sérine/thréonine kinases ou facteurs de transcription [3-5].

L'ensemble de ces résultats expérimentaux permettait de bâtir une "théorie unificatrice de la genèse des cancers" [6]. Selon cette théorie, les mutations spontanées ou induites par des cancérogènes chimiques ou des radiations, ainsi que par des rétrovirus, modifient accidentellement certains oncogènes cellulaires, qui parti- 
cipent normalement au contrôle de la prolifération cellulaire. Ces modifications conduisent à la prolifération " anarchique" des cellules cancéreuses. A cette époque, pour la majorité de ceux qui en étudiaient les mécanismes moléculaires, le cancer n'apparaissait plus comme une diversité de maladies différentes, mais comme une maladie unique caractérisée par des mécanismes communs à tous les types de tumeurs.

La première décade des oncogènes s'est terminée avec le clonage de $R B$, premier gène suppresseur de tumeur qui venait compléter harmonieusement la théorie. Alors que l'activation des produits des proto-oncogènes leur confère un rôle dominant dans la formation de la tumeur, c'est l'absence ou l'inactivation des produits des gènes suppresseurs de tumeurs - ou anti-oncogènes - qui contribue à cette formation. Les produits des gènes suppresseurs de tumeurs sont supposés participer à la transmission des signaux qui inhibent la prolifération cellulaire. Selon ce modèle, les produits de ces gènes agissent comme des freins à cette prolifération, alors que les produits des oncogènes seraient des accélérateurs.

\section{$2^{\circ}$ période: $1985-1995$. Tous coupables?}

Il serait bien difficile, aujourd'hui, de répondre à la question "Combien y at-il d'oncogènes ou de gènes suppresseurs de tumeurs ?" Au cours des dix dernières années, l'amélioration des techniques du génie génétique et les efforts consacrés à la cartographie du génome humain ont permis d'accélérer l'identification des gènes suppresseurs et des gènes de susceptibilité aux tumeurs $[4,5]$. A un rythme tout aussi soutenu s'est poursuivie l'identification de nouveaux oncogènes selon les stratégies qui avaient fait leur preuve : recherche des gènes situés aux points de translocation dans des leucémies, transfection d'ADN tumoral dans des cellules test, étude du génome de nouveaux rétrovirus et clonage des sites d'intégration de rétrovirus induisant des tumeurs en plusieurs étapes ou de virus à ADN
Au même moment, la foule des acteurs potentiels du processus de cancérisation s'est enrichie, d'une part par le regroupement de certains oncogènes par familles et le recensement de nouveaux membres, d'autre part par la recherche des protéines associées aux produits des oncogènes. On connaît au moins 50 membres dans la famille Ras, un grand nombre de kinases ou de phosphatases identifiées par une séquence consensus située au site catalytique, plus de 30 membres pour la famille du facteur de transcription Ets. Simultanément, sont entrées en scène d'autres familles de protéines qui participent au contrôle de la prolifération et de la différenciation cellulaires, et au processus de cancérisation. Il s'agit d'abord des protéines qui font partie de l'horloge cytoplasmique réglant le cycle cellulaire : cyclines, kinases dépendantes des cyclines et leurs inhibiteurs comme la p16 dont la perte ou l'inactivation a été décrite dans un grand nombre de tumeurs $\left(m / s n^{\circ} 6-7\right.$, vol. 10, p. 744) [7]. Il s'agit ensuite de certains facteurs qui règlent la traduction comme elF-4E $\left(m / s ~ n^{\circ} 7\right.$, vol. 6 , p. 688) [8], de certaines protéines du cytosquelette, de la matrice nucléaire et de certaines protéines d'adhérence. La transformation des fibroblastes est souvent accompagnée d'une synthèse réduite de plusieurs protéines se fixant à l'actine, comme la tropomyosine, la vinculine, l' $a$-actinine et l' $\alpha$ caténine. Inversement, l'expression constitutive d'un ADNc codant pour ces protéines dans des fibroblastes transformés par Ras renverse la transformation cellulaire [9]. De plus, la partie 3 ' non codante du gène de l' $\alpha$ tropomyosine supprime la prolifération et le pouvoir transformant de cellules de rhabdomyosarcome [10], résultat qui rappelle le rôle important que peuvent jouer dans la transformation cellulaire les parties non traduites des ARN messagers pour certains oncogènes, ou même des ARN non traduits comme l'ARN du gène $B A C-H 19$ [11]. Il s'agit enfin des gènes de ménage : l'étude systématique des anomalies chromosomiques dans les tumeurs solides suggère que la pression métabolique contribue à déséquilibrer progressi- vement le caryotype en fonction des nouveaux impératifs de transcription. Selon cette hypothèse, les gènes de ménage comme ceux de la synthèse nucléotidique dans les cancers colorectaux, de la glycolyse dans les cancers de l'endomètre ou du métabolisme de l'adénylate seraient des anti-oncogènes ou des oncogènes [12].

Cette accumulation de résultats a permis de révéler des associations remarquables dans les réseaux de régulation cellulaire. Ainsi a été décrit un ensemble de protéines susceptibles de se fixer aux récepteurs à activité tyrosine kinase comme le récepteur du PDGF ou de l'EGF, protéines Shc, Grb2 et Sos qui assurent la transmission du signal vers la cascade Ras [13], phospholipase C $v$, phosphatidylinositol 3-kinase et tyrosine phosphatase PTP-1D (ou Syp) qui dirigent le signal vers des voies différentes. On commence à connaître les protéines qui s'associent au facteur de transcription NFKB pour contrôler sa localisation dans la cellule [14], ou les partenaires de Myc, la protéine pl07 qui présente des analogies avec Rb et dont la fixation inhibe l'activité transcriptionnelle de Myc au cours du cycle cellulaire [15] et les protéines Max qui permettent la fixation de Myc à l'ADN. Ces protéines Max peuvent aussi former des hétéro-dimères avec les protéines Mad et Mxi-1. Les complexes Max-Mad reconnaissent les mêmes séquences d'ADN que les complexes Myc-Max, mais ils se comportent comme des inhibiteurs de la transcription, en recrutant la protéine mSin3 [16].

Cependant, au fur et à mesure que se rassemblaient autour d'eux ces parents et associés de plus en plus nombreux et difficiles à classer, le statut des oncogènes a perdu sa visibilité. Parfois c'est le produit d'un anti-oncogène qui s'associe au produit d'un oncogène pour en contrôler l'activité. C'est le cas du produit du gène suppresseur de tumeur $N F-1$, qui bloque la transmission du signal extra-cellulaire par l'intermédiaire de Ras en stimulant l'activité GTPase de la protéine Ras. La délétion du gène $N F-1$ pourrait donc avoir le même effet que les mutations du gène ras qui suppriment son activité GTPase [17]. 
Mais la classification de nombreux partenaires des oncogènes n'est pas toujours aussi facile, en partie parce qu'ils ne répondent pas à tous les critères initialement retenus pour identifier un oncogène ou un anti-oncogène. La protéine Max, abondante et stable, ne semble pas un partenaire très actif dans la transformation, tandis qu'une surexpression de Mad supprime la transformation par Myc et diminue la tumorigénicité de cellules d'astrocytome humain dans la souris nue [18]. Une surexpression de Sos et de la protéine adaptatrice Shc, après transfection, induit dans des fibroblastes NIH3T3 un phénotype transformé et tumorigène [19, 20]. Mais on n'a pas retrouvé de mutations, délétions ou translocations systématiques au gène $S H C^{\prime}$ dans des tumeurs humaines. Inversement, certains membres de la famille Ras ou de la famille Ets ont été associés à des anomalies chromosomiques alors que leur activité transformante dans le test NIH3T3 est très faible. Si certains gènes homéotiques comme les gènes Pax ou Hox 11, qui participent aux premières étapes de la morphogenèse, sont capables de transformer des cellules dans lesquelles ils sont surexprimés [21], on peut se demander si tout facteur de transcription n'est pas capable d'une activité oncogénique dans un contexte cellulaire approprié.

Parallèlement, la frontière entre oncogène et anti-oncogène est devenue floue. Dans une même famille, on peut trouver des oncogènes (ras) et un anti-oncogène (K-rev). Une simple mutation sur le gène $p 53$ le fait passer de la catégorie d'anti-oncogène à celle d'oncogène. Et l'oncogène Ela, qui transforme des cellules de souris ou de rat, agit comme un anti-oncogène sur les cellules humaines [22]. Selon le contexte cellulaire, une même protéine peut agir comme un frein ou un accélérateur : selon les facteurs de croissance présents dans le milieu de culture, la surexpression de la protéine Myc induit la prolifération ou la mort cellulaire [23].

$m / s n^{\circ} 10$, vol. 11, octobre 95

\section{Propositions pour la période à venir}

Pour une analyse systémique des régulations cellulaires

Chercher à comprendre quel peut être le rôle de la phosphorylation d'un facteur de transcription dans la prolifération ou la différenciation cellulaire, c'est chercher à établir un lien entre un événement local et le déroulement d'un processus global. Cette démarche implique l'identification des éléments du système étudié, susceptibles d'être impliqués dans ce processus, et l'étude de leurs interactions. La "carte des oncogènes" qui orne les murs de nombreux laboratoires représente une étape dans cette démarche. Cependant, on s'accordera facilement pour reconnaître que cette carte n'est pas aux régulations ce que le cycle de Krebs est au métabolisme. En effet sur ces cartes sont accumulés, dans une même cellule "mythique", les résultats expérimentaux obtenus dans des types cellulaires différents, avec des cellules situées dans des contextes différents, lignées cellulaires ou cellules primitives cultivées sur des substrats différents, parfois dans des conditions différentes de densité ou de milieu. La signification de ces cartes est aussi affectée par l'absence de référence temporelle et le caractère rudimentaire des informations spatiales. L'absence de toute donnée quantitative est enfin un handicap sérieux pour apprécier l'importance des différents niveaux de régulation dans la cellule.

Aussi est-il vraisemblable que dans dix ans ces cartes statiques, distordues et parfois trompeuses seront remplacées, au moins dans l'esprit des chercheurs, par des "films d'animation" dont on entrevoit aujourd'hui quelques séquences, quelques gros plans que nous évoquerons ici. A l'extérieur de la cellule, lorsqu'un facteur de croissance ou une hormone est présent à une concentration de $10^{-9}$ à $10^{-12} \mathrm{M}$, on peut calculer qu'en moyenne une seule molécule de ce facteur ou de cette hormone se trouve à une distance de 10 à $100 \mathrm{~mm}$ d'une cellule dont le diamètre serait $10 \mu \mathrm{m}$ ! On comprend alors que la fixation "non spé- cifique" de ces facteurs sur la matrice extra-cellulaire sera importante pour augmenter la concentration de ce facteur à proximité de la cellule [24]. La fixation du ligand sur son récepteur stabilise une conformation donnée d'une activité kinase (ou phosphatase) et/ou dans laquelle sont favorisées les interactions avec différentes protéines. L'ajout d'un phosphate sur le résidu tyrosine d'une protéine par un récepteur doué d'activité kinase nécessite in vitro quelques millisecondes alors que la transcription, si on suppose un rythme d'élongation de trente secondes par kilobase, nécessite au moins deux minutes pour le gène $c$ fos, trente minutes pour le gène $c$ ets 1 , et environ onzeheures pour un gène aussi long que celui de la dystrophine [25]. L'activité spécifique des phosphatases est 1000 fois plus élevée que celle des kinases sur des substrats artificiels [26], ce qui suggère que la cellule dispose d'un système très efficace pour contrebalancer l'action des kinases et remettre à zéro les voies qui ont été activées. En revanche, on ne connaît pas les constantes d'association, et surtout de dissociation, des protéines qui se fixent aux récepteurs ; pour une même affinité, ces paramètres essentiels peuvent varier de façon importante [27]. Ces protéines associées aux récepteurs de facteurs de croissance (Ras, Gap et les adaptateurs...) sont confinées dans un espace de quelques centaines de nanomètres près de la membrane extracellulaire et la perturbation qui les affecte va se propager vers le noyau. Les distances à franchir peuvent varier d'un facteur vingt entre les thymocytes et les fibroblastes qui ont un compartiment cytoplasmique plus volumineux. Cette perturbation peut transiter à la fois le long du cytosquelette [28], par la diffusion de protons, de calcium ou de facteurs de transcription... Ces processus ont des constantes de temps différentes et le noyau pourra recevoir une succession de signaux chronologiquement espacés. La transmission de ces signaux s'accompagne d'une internalisation continuelle des composants de la membrane cytoplasmique ; l'amplitude de ce mouvement est telle que l'équivalent de la 
surface entière d'un macrophage peut être recyclé en moins de deux heures [29], mais son importance dans la transmission des signaux n'est pas évaluée.

A l'intérieur du noyau, les phénomènes de compartimentation participent au contrôle de l'expression des gènes, et il sera important de préciser la localisation des facteurs de transcription et des kinases comme la Jun kinase Jnk dont la spécificité pourrait être guidée par leur association à un facteur de transcription [30]. La régulation de l'initiation de la transcription est un processus dynamique. Pour comprendre le rôle d'un facteur de transcription dans ce processus, on ne peut se contenter des expériences de retard sur gel ou d'empreinte sur l'ADN qui donnent simplement une information sur un équilibre de fixation. La définition des étapes de fixation et la caractérisation de leurs paramètres cinétiques seront plus informatives que la détermination d'une simple constante d'affinité. Selon la jolie formule utilisée pour décrire la régulation transcriptionnelle par l'AMPc et son récepteur dans $E$. coli, aucun parieur ne miserait sur un cheval de course simplement parce qu'il aime sa photo au repos [31] ! Cette étude de l'effet glucose dans $E$. coli et l'étude du répresseur du phage $\lambda$ sont des beaux exemples de l'importance des informations quantitatives pour la compréhension d'un processus biologique [32]. Mais, s'il est souvent difficile d'avoir ces informations quantitatives précises parce que tous les systèmes ne se prêtent pas à une telle analyse, il peut être très instructif d'évaluer, à l'échelle de la cellule, la signification des ordres de grandeur des chiffres que nous manipulons dans un tube à essais [33] . Ainsi, lorsqu'on considère des $A R N$ présents à moins de dix copies par cellule, il faut tenir compte de la variation statistique importante qui affecte ce nombre. Cette variation est peut-être à l'origine de la grande variabilité des durées des phases du cycle cellulaire lorsqu'on les mesure dans chaque cellule d'une population [34]. Cette hétérogénéité est aussi manif este lorsqu'on analyse in dants dans une lignée cellulaire ; elle suggère l'existence de processus stochastiques dans la cellule et justifie l'intérêt d'une analyse des mécanismes de régulation transcriptionnelle au niveau d'une seule cellule, et non pas seulement dans une population de cellules [35]. En paraphrasant le titre d'un éditorial de la revue Nature [36], on pourrait écrire que l'oncologie moléculaire sera une science lorsqu'une part plus grande sera consacrée à la mesure.

\section{Pour l'élaboration d'une plate-forme conceptuelle cohérente}

Aujourd'hui, et sans doute pour plusieurs années encore, la recherche de nouveaux acteurs moléculaires domine la biologie du cancer. Selon une image souvent reprise, ces acteurs constitueraient les pièces d'un gigantesque puzzle, puzzle dynamique dont on peut décrire aujourd'hui l'assemblage de certains éléments, pris deux à deux. Pour obtenir une image plus complète, pour comprendre le rôle de ces acteurs, il faudra confronter les résultats expérimentaux à des modèles. Il y a trente ans, le modèle de la transition allostérique avait suscité un essor remarquable de l'enzymologie, avant même que la structure tridimensionnelle des enzymes allostériques soit révélée. De façon surprenante, la biologie théorique et la modélisation semblent aujourd'hui absentes des préoccupations des expérimentateurs. En fait, une analyse plus fine montre que l'expérimentation et l'exploitation des résultats expérimentaux reposent sur des modèles implicites qui ne sont plus satisfaisants.

Ainsi, la disposition des chemins sur la carte des oncogènes, dirigés le plus souvent de l'extérieur de la cellule vers l'intérieur, la notion de cascade de régulation, la volonté de bloquer en amont le processus de transformation renvoient à un modèle linéaire, comme l'écoulement d'un fleuve, de la montagne vers la mer. Cette image est valide sur des segments courts; elle n'est plus correcte lorsqu'on s'intéresse aux boucles de régulations multiples et enchevêtrées qui existent dans un tissu. Dans ces régulations, le même événement peut être à la fois cause et conséquence : l'activation de certains oncogènes perturbe les interactions de la cellule avec la matrice extracellulaire, induit la production de certaines protéases qui favorisent l'invasion, ou bloque la synthèse d'une enzyme qui initie la réticulation du collagène et de l'ćlastine [37]. Mais, inversement, la présence constitutive de certaines protéases, ou le blocage de certaines molécules d'adhérence, modifie l'activité transcriptionnelle des cellules et favorise la transformation cellulaire.

Il y a une dizaine d'années, il était admis implicitement qu'à une protéine correspondait une fonction dans un organisme. On considérait alors que les oncogènes jouaient un rôle essentiel au cours du développement. Les expériences de recombinaison homologue ont remis en cause ce concept : si l'inactivation de certains oncogènes (comme c-myb, csk, Pu1/Spi-1, Tal-1, c-ret ou vav...) est létale pendant la vie embryonnaire ou se traduit par des anomalies majeures du développement, l'inactivation de relB, N-ras, c-fos ou de pim-1 n'entraîne aucun phénotype marquant, suggérant qu'existe, au cours du développement embryonnaire, une considérable redondance fonctionnelle entre les produits de plusieurs gènes. Plutôt que de chercher à décrire une "chorégraphie méticuleusement orchestrée", il nous faut chercher à comprendre comment des "règles" imprécises donnent un développement précis [38]. L'étude théorique de l'organisation biologique suggère que la complexité et la redondance fonctionnelle, non seulement améliorent la fiabilité d'un système, mais surtout permettent au système de récupérer des perturbations aléatoires pour se construire ou se reconstruire de façon nouvelle [39]. Ce concept d'organisation par le bruit mérite d'être précisé au niveau moléculaire et d'être exploité plus précisément, non seulement dans l'embryon, mais aussi dans les tissus adultes et dans les tumeurs. Il est possible que les réseaux de régulation deviennent plus rigides dans les cellules cancéreuses, à la suite des amplifications et des délétions chromosomiques, et que cette rigidité soit exploitable à des fins thérapeutiques. 


\section{Oncogènes anti-oncogènes et thérapie}

Les vingt années qui s'achèvent ont été passionnantes pour ceux qui ont participé à la recherche des mécanismes moléculaires du cancer. Les connaissances accumulées ont ouvert la voie à de nombreux travaux consacrés à la mise en œuvre de protocoles thérapeutiques. Dans ces protocoles sont visées aussi bien les cellules cancéreuses que les cellules du stroma tumoral, avec une gamme d'outils de plus en plus large : agents pharmacologiques inhibant spécifiquement des activités enzymatiques (kinases, protéases) ou des interactions protéineprotéine dans les réseaux de signalisation [40], oligonucléotides antisens ou vecteurs viraux destinés à tuer les cellules cancéreuses ou à les "rééduquer ". L'hypothèse sous-jacente à ces protocoles est la suivante : si un maillon faible, manquant ou dominant, a été identifié dans un système biologique en fonctionnement pathologique, on devrait obtenir un résultat thérapeutique au moyen d'un renforcement, d'une substitution ou d'une inhibition du maillon identifié [41]. Les difficultés de l'entreprise sont liées à la redondance des mécanismes de régulation, à la multiplicité des rôles des acteurs moléculaires de ces régulations et surtout à l' " homéostasie pathologique", qui fait que le système est capable d'annuler ou de limiter l'action souhaitée [41]. Tout en poursuivant l'identification de nouveaux oncogènes et anti-oncogènes, il faudrait consacrer autant d'efforts à la description cinétique et spatiale des différents niveaux de régulation dans les systèmes vivants, à l'étude de leurs interactions et à leur modélisation pour pouvoir définir des stratégies thérapeutiques efficaces

\section{Remerciements}

Je remercie Michel Bomens, Henri Buc, Jean Coll, Véronique Fafeur, Michel Morange, Christophe Queva, Jean-Claude Salomon, Dominique Stehelin et les arbitres de médecine/sciences pour leurs encouragements et leurs critiques après la lecture de ce manuscrit.

\section{RÉFÉRENCES}

1. Stehelin D. Les oncogènes cellulaires clés de la cancérogenèse. médecine/sciences $1985 ; 1: 12-6$

2. Bishop JM. Oncogenes. Sci Am 1982 ; 246 : $68-78$

3. Kahn A. La saga des oncogènes. médecine/sciences $1985 ; 1$ : 10-1.

4. Kahn A. Gènes et cancers en 1988. méderine/sriences $1988 ; 4: 606-7$.

5. Tambourin $P$. Oncogènes et oncogenèse.médecine/sciences $1990 ; 6: 340-2$.

6. Stehelin D. Dissection moléculaire des cancers. Bull Inst Pasteur 1988 ; 86 : 225-42.

7. Hunter T, Pines, J. Cyclins and cancer II : cyclin $\mathrm{D}$ and $\mathrm{CDK}$ inhibitors come of age. Cell $1994 ; 79$ : 573-82.

8. Sonenberg N. Translation factors as effectors of cell growth and tumorigenesis. Curr Biol 1993 ; 5 : 955-60.

9. Prasad GL, Fuldner, RA, Cooper, HL. Expression of transduced tropomyosin 1 cDNA suppresses neoplastic growth of cells transformed by the ras oncogene. Proc Nall Acad Sci USA 1993 ; 90 : 7039-43.

10. Rastinejad F, Conboy, MI, Rando, TA Blau HM. Tumor suppression by RNA from the 3 untranslated region of $\alpha$-tropomyosin. Cell $1993 ; 75$ : 1107-17.

11. Hao $Y$, Crenshaw $T$, Moulton $T$, Newcomb E, Tycko B. Tumour-suppressor activity of H19 RNA. Nature $1993 ; 365: 764-7$.

12. Dutrillaux B. Les anomalies chromosomiques dans les tumeurs solides : quelle signification? médecine/sciences $1991 ; 7: 10-2$.

13. Chardin P. Protéines Ras et transmission des signaux mitogènes. möderine/sciences 1994 ; 10 : 657-64.

14. Liou HC, Baltimore, D. Regulation of the NF- $\kappa \mathrm{B} / \mathrm{rel}$ transcription factor and IkB inhibitor system. Curr Op Cell Biol 1993; 5 : $477-87$

15. Gu W, Bhatia K, Magrath IT, Dang CV, Dalla-Favera R. Binding and suppression of the Myc transcriptional activation domain by p107. Srience $1994 ; 264: 251-4$.

16. Ayer D, Lawrence QA, Eisenman RN. Mad-Max transcriptional repression is mediated by ternary complex formation with mammalian homologs of yeast repressor Sin3. Cell $1995 ; 80: 767-76$.

17. Bollag G, McCormick F. NF is enough of GAP. Nature $1992 ; 356: 663-4$.

18. Chen J, Willingham $T$, Margraf LR Schreiber-Agus N, DePinho RA, Nisen PD. Effects of the MYC oncogene antagonist, MAD, on proliferation, cell cycling and the malignant phenotype of human brain tumour cells. Nature Med 1995; 1:638-43.
19. Pelicci G, Lanfrancone L, Grignani F, McGlade I, Cavallo F, Forni G, Nicoletti I, Grignani F, Pawson T, Pelicci PG. A novel transforming protein (Shc) with an $\mathrm{SH} 2$ domain is implicated in mitogenic signal transduction. Cell $1992 ; 70: 93-104$

20. Egan SE, Giddings BW, Brooks MW, Buday $L$, AM S, Weinberg RA. Association of Sos Ras exchange protein with Grb2 is implicated in tyrosine kinase signal transduction and transformation. Nature $1993 ; 363$ : 45-51.

21. Maulbecker CC, Gruss P. The oncogenic potential of Pax genes. EMBOJ 1993 ; 12 : 2361-7.

22. Frisch SM. Ela induces the expression of epithelial characteristics. J Cell Biol 1994 ; $127: 1085-96$

23. Piechaczyk M. Myc : le paradoxe d'un oncogène induisant la mort cellulaire programmée. méderine/sciences $1992 ; 8: 586-7$.

24. Sumper M, Berg E, Wenzl S, Godl K. How a sex hormone might act at a concentration below $10-16 \mathrm{M}$. EMBO J 1993 ; 12 : 831-6.

25. Shermoen AW, O'Farrell P. Progression of the cell cycle through mitosis leads to abortion of nascent transcripts. Cell 1991 ; $67: 303-10$.

26. Fischer EH, Charbonneau H, Tonks NK. Protein tyrosine phosphatases: a diverse family of intracellular and transmembrane enzymes. Science $1991 ; 253$ : 401-6.

27. Williams A. Out of equilibrium. Nature $1991 ; 352: 473-4$

28. Forgacs G. On the possible role of cytoskeletal filamentous networks in intracellular signaling: an approach based on percolation. I Cell Sri 1995 ; 108 : 2131-43.

29. Steinman RM, Mellman IS, Muller WA Cohn ZA. Endocytosis and the recycling of plasma membrane. I Cell Biol 1983; 96 : 1-

30. Karin M. Signal transduction from the cell surface to the nucleus through the phosphorylation of transcription factors. Curr Op Cell Biol 1994 ; 6 : 415-24.

31. Buc $H$. Mechanism of activation of transcription by the complex formed between cyclic-AMP and its receptor in Escherichia coli. Biochem Soc Trans 1986 ; 14 : 196-9.

32. Maddox J. Towards more measurement in biology. Nature 1994 ; 368 : 95.

33. Albrecht-Buehler G. In defense of "nonmolecular" cell biology. Int Rev Cytol 1990; $120: 191-241$.

34. Collyn M, Malaise EP, Valleron AJ. Time lapse cinematographic studies of cell cycle and mitosis duration. Exp Cell Res 1977 ; 106: $405-7$

35. White MRH, Masuko M, Amet L, Elliott G, Braddock M, Kingsman AJ, Kingsman SM. Real-time analysis of the transcriptional 
regulation of HIV and hCMV promoters in single mammalian cells. J Cell Sci $1995 ; 108$ $441-55$.

36. Maddox J. Is molecular biology yet a science? Nature $1992 ; 355: 201$

37. Contente S, Kenyon K, Rimoldi D, Friedman RM. Expression of gene $r g$ is associated with reversion of NIH 3 T3 transformed by LTR-c-H-ras. Science $1990 ; 249: 796-8$

38. Stryker MP. Precise development from imprecise rules. Science 1994 ; 263 : 1244-5.

39. Atlan H. Entre le cristal et la fumée. Essai sur l'organisation du vivant. Paris : Le Seuil, 1979.

40. Gibbs JB, Oliff, A. Pharmaceutical research in molecular oncology. Cell 1994 ; $79: 193-8$

41. Bernard-Weil E. Les thérapeutiques bipolaires. Rev Intern System 1988; 2 : 399-415.

\section{Bernard Vandenbunder}

Laboratoire d'oncologie moléculaire, Cnrs Ura 1160, Institut Pasteur de Lille, 1, rue Calmette, 59019 Lille Cedex, France.

\section{Summary}

A new view on oncogenes, twenty years after the discovery of c-src

The discovery of the first oncogene in 1975 and the experimental data accumulated during the following years allowed the establishment of an "unifying theory" for the development of cancers. According to this theory, cancers result from a deregulated expression or from a mutation of a limited number of genes, called oncogenes. After the recruitment of new members, relatives and associates in the club, the position of oncogenes and anti-oncogenes became more and more fuzzy. In order to understand the roles of these molecular actors, it is important to put them into their context, to undertake the kinetic description of their interactions and to take into account quantitative considerations. One should also elaborate a new paradigm in order to be able to organize and predict experimental results. 